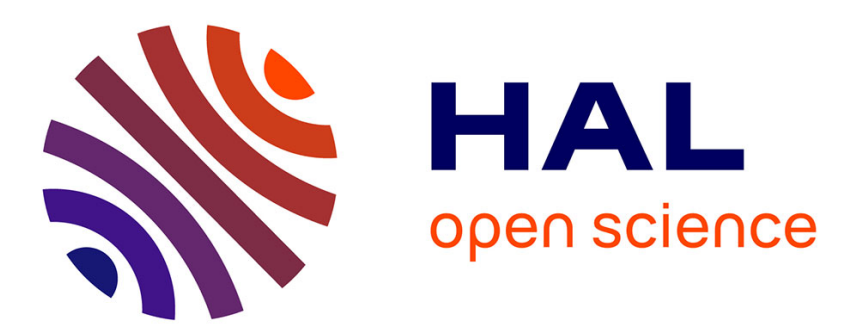

\title{
Modification of the spontaneous seat-to-stand transition in cycling with bodyweight and cadence variations
}

\author{
Bruno Watier, Antony A Costes, Nicolas A Turpin
}

\section{To cite this version:}

Bruno Watier, Antony A Costes, Nicolas A Turpin. Modification of the spontaneous seat-to-stand transition in cycling with bodyweight and cadence variations. Journal of Biomechanics, 2017, 63, pp.61-66. 10.1016/j.jbiomech.2017.08.003 . hal-01584024

\section{HAL Id: hal-01584024 \\ https://hal.laas.fr/hal-01584024}

Submitted on 8 Sep 2017

HAL is a multi-disciplinary open access archive for the deposit and dissemination of scientific research documents, whether they are published or not. The documents may come from teaching and research institutions in France or abroad, or from public or private research centers.
L'archive ouverte pluridisciplinaire HAL, est destinée au dépôt et à la diffusion de documents scientifiques de niveau recherche, publiés ou non, émanant des établissements d'enseignement et de recherche français ou étrangers, des laboratoires publics ou privés. 
Modification of the spontaneous seat-to-stand transition in cycling with bodyweight and cadence variations

Bruno Watier ${ }^{1, *}$, Antony Costes ${ }^{2}$, Nicolas A. Turpin ${ }^{3,4}$

${ }^{1}$ LAAS, CNRS, University of Toulouse, France

${ }^{2}$ PRISSMH- LAPMA, University of Toulouse, France

${ }^{3}$ Department of Neuroscience, University of Montreal, Qc, Canada

${ }^{4}$ Center for Interdisciplinary Research in Rehabilitation of Greater Montreal (CRIR), Montreal, QC, Canada

*: corresponding author:

\section{Bruno Watier}

LAAS - CNRS - 7, av du colonel Roche - 31400 Toulouse - France

E-mail address: bruno.watier@univ-tlse3.fr

Phone number: +33 622075004

Fax number: +33561336300 


\section{Abstract:}

When a high power output is required in cycling, a spontaneous transition by the cyclist from a seated to a standing position generally occurs. In this study, by varying the cadence and cyclist bodyweight, we tested whether the transition is better explained by the greater power economy of a standing position or by the emergence of mechanical constraints that force cyclists to stand.

Ten males participated in five experimental sessions corresponding to different bodyweights (80\%, 100\%, or 120\%) and cadences (50 RPM, 70 RPM, or 90 RPM). In each session, we first determined the seat-to-stand transition power (SSTP) in an incremental test. The participants then cycled at $20 \%, 40 \%, 60 \%, 80 \%, 100 \%$, or $120 \%$ of the SSTP in the seated and standing positions, for which we recorded the saddle forces and electromyogram (EMG) signals of eight lower limb muscles. We estimated the cycling cost using an EMG cost function (ECF) and the minimal saddle forces in the seated position as an indicator of the mechanical constraints.

Our results show the SSTP to vary with respect to both cadence and bodyweight. The ECF was lower in the standing position above the SSTP value (i.e., at 120\%) in all experimental sessions. The minimal saddle forces varied significantly with respect to both cadence and bodyweight.

These results suggest that optimization of the muscular cost function, rather than mechanical constraints, explain the seat-to-stand transition in cycling. 


\section{Introduction}

The pedaling movement can be performed in either of two positions: seated or standing (Ryschon et al., 1991). However, the criteria leading to a cyclist’s spontaneous transition from one position to the other are unclear.

Although the standing position allows higher crank-power outputs compared to the seated position (McLester et al., 2004; Millet et al., 2002; Reiser et al., 2002), this position is not always chosen, particularly at low power outputs. Moreover, spontaneous transition from a seated to a standing position is generally observed before maximum power is reached in the seated position (Poirier et al., 2007). Based on previous studies, at least two hypotheses can be formulated to explain this spontaneous transition. First, the cycling movement may be associated with a cost function whose value is reduced when the transition is made (Costes et al., 2017; Poirier et al., 2007; Turpin et al., 2016). Alternatively, the mechanical constraints that arise at a high power output may lead the cyclist to transition from a seated to a standing position (Costes et al., 2015).

In both positions, the magnitude of the mechanical variables (e.g., joint torques), the muscle activation levels, and the metabolic energy expenditure increase with power output (Li et al., 1998; McDaniel et al., 2002, 2014). However, joint torques and muscle activation levels increase at a higher rate in the seated position but have lower baseline values, i.e., lower electromyogram (EMG) levels and torque when the power output is zero (Turpin et al., 2016). As such, it has been suggested that the standing position becomes advantageous only at high power outputs (Turpin et al., 2016). This conclusion is also supported by the results of Hansen et al. (2008) who observed that the standing position extended the time to exhaustion compared to that of the seated position, but only at high levels of power output (i.e., above $94 \%$ of the power output at maximal oxygen uptake). In the same vein, Poirier et al. (2007) showed that a moment cost function (MCF, Gonzalez et al. , 1989) based on the sum of the lower limb joint 
torques was lower in a spontaneously chosen position at any given power output, except those very close to the power output at which the transition was made. MacIntosh et al. (2000) proposed that the averaged EMG activity of several muscles may also represent the mechanical cost of the lower limbs. Along the same lines, Turpin et al. (2016) showed that the integrated EMG signals of the lower limb muscles in the standing position were less than or equal to those in the seated position at high power outputs (i.e., > $500 \mathrm{~W}$ ), which suggests a lower "EMG cost" in the standing position at these power outputs.

In support of the second hypothesis, our team showed that when power output increases, the forces applied on the pedals create reaction forces that tend to lift the upper body and decrease the forces applied by the trunk on the saddle (Costes et al., 2015). We observed the occurrence of spontaneous transition when the minimal saddle reaction forces were close to zero (i.e., 1 $\mathrm{N} / \mathrm{kg}$ ). The spontaneous seat-to-stand transition power (SSTP) also coincides with the appearance of strategies for counteracting the elevation of the trunk (handlebars and pedal pulling), and we hypothesized that the spontaneous SSTP was related to the emergence of these mechanical constraints.

In this study, we further test these two hypotheses by studying the power output at which subjects with various bodyweights and cadences spontaneously transition to the standing position (i.e., SSTP). We hypothesized that if the transition was linked to the emergence of upward forces not fully offset by the subject's bodyweight, then varying the bodyweight or cadence might affect SSTP but not the minimal saddle forces at the time of transition. On the other hand, if the transition is made to minimize EMG cost, then we expect the ECF to be lower in the seated position (compared to the standing position) below the SSTP and correspondingly higher above the SSTP for all cadences and bodyweights. Such a finding would strongly support the first hypothesis as cadence is known to have large effects on lower limb muscles activity and coordination (Baum and Li, 2003). 


\section{Method}

\section{Participants}

Ten healthy males (age: $21.2 \pm 2.1$ years, body mass: $72.9 \pm 5.3 \mathrm{~kg}$, height: $1.79 \pm 0.07$ meters) volunteered for the study. Participants were non-cyclists and belonged to categories 4 and 5 from the Ansley and Cangley classification (2009). Each participant was informed of the experimental procedure and signed an informed consent form prior to the study. They were asked to avoid high-intensity or exhaustive exercise at least 48 hours before each laboratory sessions. The study was conducted in accordance with the declaration of Helsinki and was approved by the University of Toulouse ethical committee.

\section{Experimental protocol}

The cycling tests were performed on a frictionless electromagnetic cycling ergometer Excalibur (LODE, Groningen, Netherlands). Positioning on the ergometer was standardized (see below).

Each subject performed five experimental sessions in a random order (Fig. 1A). In two sessions the subjects performed the tests either with their bodyweight (BW) decreased by $20 \%$ (80\% BW) or increased by $20 \%$ (120\% BW) while the cadence was fixed to 70 RPM. In three other sessions subjects performed the tests at a fixed cadence of 50, 70 or 90 RPM without weight modification (100\% BW). A rest period of two days was imposed between each session to minimize the effects of fatigue.

Each session consisted in two tests. Test \#1: After a five-minute warm-up at $100 \mathrm{~W}$, the subjects completed a series of 20 s stages, starting at a power of $200 \mathrm{~W}$ and incremented by $25 \mathrm{~W}$ for each subsequent stage. These increments allowed a precise estimation of the transition power. Recovery periods of $40 \mathrm{~s}$ at $50 \mathrm{~W}$ were implemented between each of the $20 \mathrm{~s}$ stages to avoid fatigue (Fig. 1B). SSTP was defined as the power output at which participants rose from the saddle during at least $10 \mathrm{~s}$ (Costes et al. 2015). 
Test \#2: After five minute of rest, the participants performed twelve randomized trials at power outputs corresponding to $20,40,60,80,100$ or $120 \%$ of SSTP in the seated or the standing position. Each pedaling trial began with a stabilization period of $10 \mathrm{~s}$ at the target power output, followed by $10 \mathrm{~s}$ of data recording. Three minutes of passive rest were given between each trials.

A screen placed in front of the participants provided a visual feedback of the pedaling cadence who were instructed to maintain it at the required value.

\section{Loading and weight support}

In the $120 \%$ BW condition, a weight corresponding to $20 \%$ of the participant total bodyweight was added to a backpack rigidly fixed to the trunk. For the $80 \% \mathrm{BW}$ condition the same weight was subtracted by applying a constant upward force through a harness fixed to the trunk by a non-compliant rope and two frictionless pulleys attached to weights (Fig. 1C).

\section{Standardized Positioning}

The seat tube angle was $73^{\circ}$ and the crank length $0.17 \mathrm{~m}$. The pedal cleat was positioned under the first metatarsal bone. The saddle height was adjusted to obtain a $150^{\circ}$ knee angle during full leg extension (Bini et al., 2011). Drop and reach lengths were adjusted according to torso and arm lengths (see de Vey Mestdagh, 1998). Positioning of the hands on the handlebar was left up to the participant (flat handlebar, width: $0.7 \mathrm{~m}$ ).

\section{Data acquisition}

The 3D forces components applied to the saddle tube were recorded from three tubular sensors (SENSIX, Poitiers, France) at $1 \mathrm{kHz}$. The sensors were factory-calibrated and their offsets were adjusted before each experiments (Costes et al., 2015). 
Surface EMGs were recorded from 8 muscles of the right side of the body : tibialis anterior (TA), soleus (SOL), gastrocnemius lateralis (GL), vastus lateralis (VL), vastus medialis (VM), rectus femoris (RF), biceps femoris (BF) and gluteus maximus (Gmax). Prior to electrode application, the skin was shaved and cleaned with alcohol to minimize impedance. Each wireless electrode (MiniWave, Cometa, Bareggio, Italy) was placed according to the recommendations of Surface EMG for Non-Invasive Assessment of Muscles (SENIAM). Electrodes were secured with adhesives tapes before recordings. EMG signals were amplified $(\times 1000)$ and recorded at $1 \mathrm{kHz}$.

Kinetics and EMG data were synchronized using Nexus software 1.7.1 (Vicon, Oxford, UK).

\section{Data reduction and analysis}

Saddle forces data were low-pass filtered at $8 \mathrm{~Hz}$ (zero lag, $4^{\text {th }}$ order Butterworth). The minimum vertical force over all cycles applied on the saddle by the cyclist in each trial of the second test was extracted for further analysis (one minima per trial). Saddle forces were then normalized by the subject actual bodyweight (i.e., $80 \%, 100 \%$ or $120 \%$ of the subject's initial body weight depending on the condition) to express forces in a dimensionless form (Villeger et al., 2012).

EMG signals were bandpass filtered (zero lag, $4^{\text {th }}$ order Butterworth) between 20 and $400 \mathrm{~Hz}$ and then rectified. For each trial in the second test, the EMG of each muscle was integrated (trapezoidal method) over the number of cycles available ( $~ 8$ to 15 cycles) and divided by the number of cycle to define the iEMG. The pedaling cycles were identified by a trigger signaling the lowest right pedal position. The iEMG value was then normalized by the mean iEMG across all trials of the second test (Turpin et al. 2016).

The EMG Cost Function (ECF) was defined as (MacIntosh et al., 2000): 
$\mathrm{ECF}=\frac{1}{m} \sum_{k=1}^{m} \mathrm{iEMG}_{k}$

where $i E M G_{k}$ represents normalized $i E M G$ value for the $k^{\text {th }}$ muscle and $m(=8)$ is the number of muscles recorded.

\section{Statistics}

Normality and homogeneity of variances were verified using Shapiro-Wilk and Levene’s tests respectively. Repeated measures ANOVAs were used to analyse the effect of cadence and bodyweight on SSTP, crank-torque and ECF and to compare saddle forces at 100\% SSTP. Posthoc analyses were performed using Bonferroni's method. The relation between power output and ECF or the saddle forces was assessed using Pearson's $r$ for each subject independently. The effect of the position on the slope and y-intercept in the relation between power output and ECF was assessed using paired t-tests. The effect of the position and bodyweight on the slopes and y-intercepts in the relation between power-output and saddle forces were assessed using Repeated measures ANOVAs. The effect of power output on saddle forces at $100 \%$ SSTP was assessed using Friedman ANOVAs. Statistical significance was set at $\mathrm{p}<0.05$.

\section{Results}

\section{SSTP}

Fig. 2 shows the SSTP values obtained in each session (group data). SSTP significantly increased with cadence $(\mathrm{p}<0.001$; Fig. $2 \mathrm{~A})$. Post-hoc results showed differences between 90 RPM and 50 RPM $(\mathrm{p}<0.001)$ and between 50RPM and 70 RPM $(\mathrm{p}<0.001)$.

A significant effect of the bodyweight was found ( $p=0.016-$ Fig. 2B). Post-hoc analysis revealed differences between $120 \%$ and $80 \%$ BW ( $\mathrm{p}=0.048$ ) and between $100 \%$ and $80 \%-\mathrm{BW}(\mathrm{p}=0.008)$. Crank-torque at SSTP decreased with increasing cadence ( $<<0.001$; Fig. 2C) and was affected by the bodyweight ( $p=0.007$; Fig. 2D). 
ECF increased linearly with power-output in all experimental conditions (Fig. 3) with R-values of $0.99 \pm 0.02$ and $0.92 \pm 0.11$ in the seated and standing position respectively (R-values computed over all conditions and subjects).

The slopes were $~ 2-3$ times greater in the seated position than in the standing position in all experimental conditions $(\mathrm{p}<0.001)$. Seated/standing ratios ranged between $1.80 \pm 0.55$ (50RPM-100\% BW) to $2.74 \pm 1.12$ (70 RPM-120\% BW). Conversely, y-intercepts were 1.510 times greater in the standing position in all experimental conditions $(\mathrm{p}<0.001)$. Standing/seated ratios ranged between $1.5 \pm 10.9$ (50 RPM-100\% BW) and $11.4 \pm 19.1$ (70 RPM- 80\% BW).

ECF significantly increased with power-output ( $<<0.001$; Fig. 3). Interaction was significant in all conditions $(p<0.001)$. ECF was significantly higher in the standing position at $20 \%$ and $40 \%$ SSTP $(\mathrm{p}<0.05)$ in all conditions and higher in the standing position at $60 \%$ SSTP in the 90 RPM- 100\% SSTP and 70 RPM-120\% SSTP conditions ( $p<0.05)$. Conversely, ECF was higher in the seated position at $120 \%$ SSTP $(\mathrm{p}<0.05)$ in all conditions.

\section{Minimal saddle vertical forces}

Variances for the saddle forces were not homogeneous $(\mathrm{p}<0.001)$. Minimal saddle forces decreased with power-output in all conditions ( $<<0.001-$ Fig. 4). Relations between power output (in \% SSTP) and saddle forces were linear with R-values of $-0.94 \pm 0.09$ over all subjects and conditions. Y-intercepts were similar between all conditions (i.e., = $38.8 \pm 9.4 \%$ BW on average; $p=0.249)$. The slopes varied between the conditions $(p=0.018)$. Post-hoc analysis showed differences between 70 RPM-100 BW and 90 RPM-100 BW (i.e., -0.34 \pm 0.07 and $0.25 \pm 0.09 \mathrm{BW}$ per \% SSTP respectively; $\mathrm{p}=0.031$ ). Values of saddle forces were further compared at 100\% SSTP (Fig. 4C). An effect of the cadence was found ( $p=0.0143$ ) with 
differences between 50 and 70 RPM $(\mathrm{p}<0.031)$. An effect of the powe output was found ( $p=0.042)$ with differences between 80\% BW and 120\% BW ( $p=0.046)$.

\section{Discussion}

In this study, we describe for the first time the effect of pedaling cadence and bodyweight on the seat-stand transition in cycling. Our study objective was to test whether the spontaneous seat-to-stand transition in cycling was made to minimize the EMG cost function or in response to mechanical constraints. As we discuss below, our data support the first hypothesis.

Is the sit-stand transition influenced by mechanical constraints?

Costes et al. (2015) suggested that in untrained participants spontaneous seat-to-stand transitions could be triggered by the vertical forces applied on the saddle. The upward forces arising when the power output increases tend to lift the upper body and it has been supposed that when these forces reach a level sufficient to compensate for the bodyweight, transition occurs. The emergence of strategies for counteracting these upward forces at the SSTP (i.e., pedal and handlebar pulling) support this interpretation (Costes et al. 2015). Subjects did not received any instructions about their pedaling technique. In this study, we found minimal forces applied on the saddle to decrease with power output and reach very low levels at the SSTP (Fig. 4). However, we found these levels to vary significantly at the SSTP, whereas we had expected similarity in these levels, which led to the rejection of the second hypothesis. Although these forces may represent constraints on the cyclist and have some influences on the seat-stand transition, they are not predictive of the power at which the transition occurs.

We observed that SSTP did not vary significantly between 100\% BW and 120\% BW (Fig. 2). This result was surprising because sustaining a heavier BW a priori requires more EMG activity in the standing position, whereas no major changes are expected in the seated position. We observed some differences consistent with these expectations in the relative positions of the 
standing and seated ECF lines in the two conditions, notably at low power (Figs. 3B and 3D). However, an SSTP level of $500 \mathrm{~W}$ occurred at both 100\% BW and 120\% BW. As such, we hypothesized that lower-limb EMG activity was not greatly influenced by the additional weight. One hypothesis could be that the added weights were redistributed to the handlebars via the upper limbs and only minimally influenced the activity of the lower-limb muscles. This possibility is suggested by Fig. 4, in which the variance of the saddle forces decrease with increasing output power. We can interpret the high variability in these forces at low powers as reflecting the presence of redundancy (Bernstein, 1967; Scholz et al., 1999), in the sense that different combinations of forces applied to the pedals, saddle, and handlebars could have been used at these power levels. Then, it is possible that adding weight caused a redistribution of weight to the handlebars, thereby limiting its influence on the lower-limb muscles.

Can other mechanical constraints explain the seat-stand transition? Because we observed SSTP and crank torque to vary with the experimental conditions (Fig. 2), power output and crank torque are not likely to trigger the transition. However, we can ask whether the SSTP might correspond to a certain percentage of the maximal power output or the maximal crank torque attainable by the subject rather than to their absolute values. Inertia of the segments and mechanical effectiveness, which influence pedal forces, may have varied with our experimental conditions (e.g., Ettema et al., 2009). As such, it could be verified in a future study whether or not pedal forces were similar between conditions and trigger the transition.

\section{The standing position reduces the ECF above the SSTP}

The results confirm our first hypothesis - above the SSTP in all conditions, the ECF was significantly lower in the standing position compared to the seated position-which suggests that the transition to the standing position is made to reduce muscular cost. The ECF increased linearly with power output in both the seated and standing positions and the functions intersected close to the SSTP value. The intersection of the cost functions suggests that there is 
a power value at which the cost of cycling is similar in both positions and that above this value, the standing position is more economical in terms of EMG. Conversely, below this power value, the seated position is more economical. This intersection point actually occurred in all conditions slightly below the SSTP (i.e., $92.4 \pm 27.2 \%$ SSTP across all subjects and conditions) and corresponded to very different power outputs depending on the conditions. One explanation for this would be that the transition was made only when the difference in terms of the EMG cost between these positions became significant. However, despite the empirical evidence of congruence between the ECF values of the two positions and the SSTP, we cannot rule out the possibility that the cost function does not precisely reflect the cycling cost.

The obtained results are in agreement with optimal control theories that consider human movement to be performed in accordance with a principle of cost function minimization (Todorov et al., 2002). Several studies have shown that the level of muscular activity can be accurately perceived by the central nervous system (Nelson, 1983; Nubar et al., 1961; Windhorst, 2007). The results of our study suggest that the ECF can be predicted for movements not yet performed. More precisely, when subjects performed the first test, they chose to stand at a power output that corresponded approximately to when the ECF was lower than in the seated position, which suggests the ability to predict that the ECF would be lower if they chose to stand at this power value. This conclusion might be surprising, given that the subjects were not cycling experts and given the variety of conditions in which the tests were performed. Overall, our results suggest that the choice of a given coordination mode in cycling is based on optimization principles.

\section{Conclusions}

In this study, we showed that the SSTP is affected by bodyweight and cadence, and that the optimization of muscular effort represents a robust predictor of the SSTP in cycling. In addition, the study results suggest that individuals with no more than average cycling experience have 
the ability to estimate the cost associated with a particular position in terms of muscular effort as they spontaneously chose to adopt the standing position when the ECF was lower than that in the seated position.

\section{Acknowledgements}

The authors are grateful to Remi Gouze for his help in data collection. Part of this work was supported by European Research Council for the project Actanthrope (ERC-ADG340050). 


\section{REFERENCES}

Ansley, L., and Cangley, P. (2009). Determinants of “optimal” cadence during cycling. European Journal of Sport Science 9, 61-85.

Baum, B.S. and Li, L., (2003). Lower extremity muscle activities during cycling are influenced by load and frequency. Journal of Electromyography and Kinesiology 13(2), 181-190.

Bernstein, N. (1967). The coordination and regulation of movements. London: Pergamon Press.

Bini, R., Hume, P.A. and Croft, J.L. (2011). Effects of bicycle saddle height on knee injury risk and cycling performance. Sports medicine 41(6), 463-476.

Costes, A., Turpin, N.A., Villeger, D., Moretto, P., and Watier, B. (2015). A reduction of the saddle vertical force triggers the sit-stand transition in cycling. Journal of Biomechanics 48, 2998-3003.

Costes, A., Turpin, N.A., Moretto, P., and Watier, B. (2017). Change From Seated To Standing Cycling Position With Increasing Power Is Associated With A Minimization Of Cost Functions. Journal of Sports Sciences (in press).

Ettema, G., Lorås, H. and Leirdal, S. (2009). The effects of cycling cadence on the phases of joint power, crank power, force and force effectiveness. Journal of electromyography and kinesiology 19(2), e94-e101.

Gonzalez, H., and Hull, M.L. (1989). Multivariable optimization of cycling biomechanics. Journal of Biomechanics 22, 1151-1161.

Hansen, E.A., and Waldeland, H. (2008). Seated versus standing position for maximization of performance during intense uphill cycling. Journal of Sports Sciences 26, 977-984.

Li, L., and Caldwell, G.E. (1998). Muscle coordination in cycling: effect of surface incline and posture. Journal of Applied Physiology 85, 927-934.

MacIntosh, B.R., Neptune, R.R., and Horton, J.F. (2000). Cadence, power, and muscle activation in cycle ergometry. Medicine and Science in Sports and Exercise 32, 1281-1287.

McDaniel, J., Durstine, J.L., Hand, G.A., and Martin, J.C. (2002). Determinants of metabolic cost during submaximal cycling. Journal of Applied Physiology 93, 823-828.

McDaniel, J., Behjani, N.S., Elmer, S.J., Brown, N.A., and Martin, J.C. (2014). Joint-Specific Power-Pedaling Rate Relationships During Maximal Cycling. Journal of Applied Biomechanics 30, 423-430.

McLester, J.R., Green, J.M., and Chouinard, J.L. (2004). Effects of standing vs. seated posture on repeated wingate performance. The Journal of Strength \& Conditioning Research 18, 816820.

Millet, G.P., Tronche, C., Fuster, N., and Candau, R. (2002). Level ground and uphill cycling efficiency in seated and standing positions. Medicine and Science in Sports and Exercise 34, $1645-1652$. 
Nelson, W.L. (1983). Physical principles for economies of skilled movements. Biological Cybernetics 46, 135-147.

Nubar, Y., and Contini, R. (1961). A minimal principle in biomechanics. The Bulletin of Mathematical Biophysics 23, 377-391.

Poirier, E., Do, M., and Watier, B. (2007). Transition from seated to standing position in cycling allows joint moment minimization. Science \& Sports 22, 190-195.

Reiser, R., Maines, J., Eisenmann, J., and Wilkinson, J. (2002). Standing and seated Wingate protocols in human cycling. A comparison of standard parameters. European Journal of Applied Physiology 88, 152-157.

Ryschon, T.W., and Stray-Gundersen, J. (1991). The effect of body position on the energy cost of cycling. Medicine \& Science in Sports \& Exercise 23, 949-953.

Scholz, J.P., and Schöner, G. (1999). The uncontrolled manifold concept: identifying control variables for a functional task. Experimental Brain Research 126, 289-306.

Todorov, E., and Jordan, M.I. (2002). Optimal feedback control as a theory of motor coordination. Nat Neurosci 5, 1226-1235.

Turpin, N.A., Costes, A., Moretto, P., and Watier, B. (2016). Can muscle coordination explain the advantage of using the standing position during intense cycling? Journal of Science and Medicine in Sport.

de Vey Mestdagh, K. (1998). Personal perspective: in search of an optimum cycling posture. Applied Ergonomics 29, 325-334.

Villeger, D., Delattre, N., Watier, B., and Moretto, P. (2012). Froude and Strouhal dimensionless numbers to study human gait: an experimental approach. Computer Methods in Biomechanics and Biomedical Engineering 15, 189-190.

Windhorst, U. (2007). Muscle proprioceptive feedback and spinal networks. Brain Research Bulletin 73, 155-202. 


\section{A Experimental sessions}

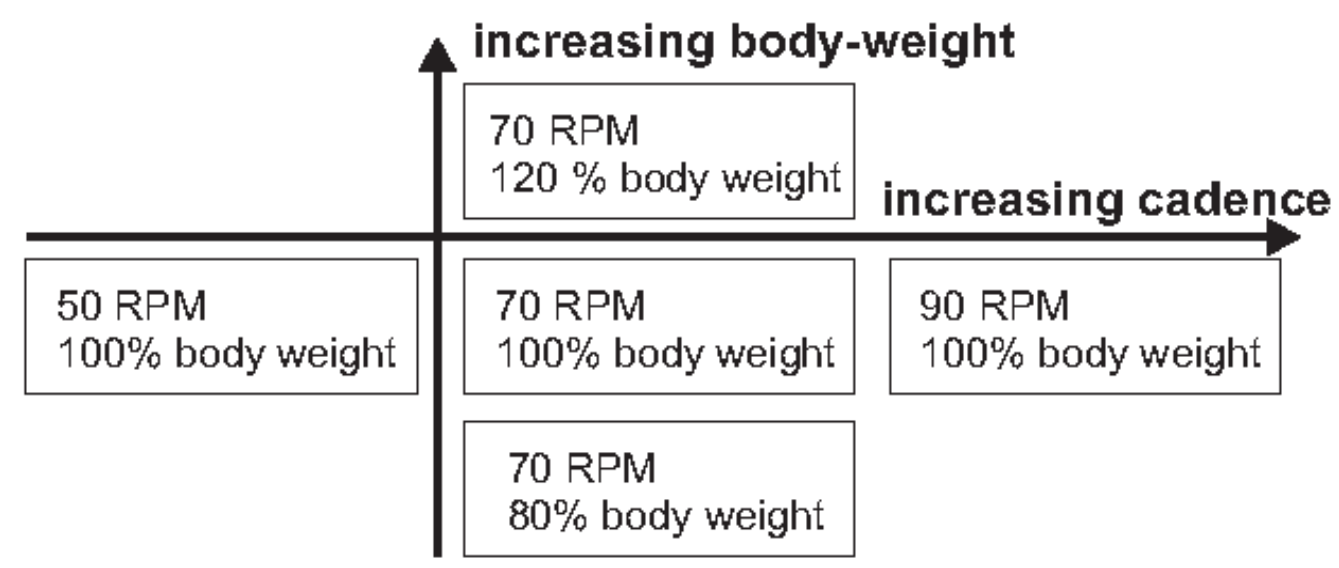

B Protocol for each session

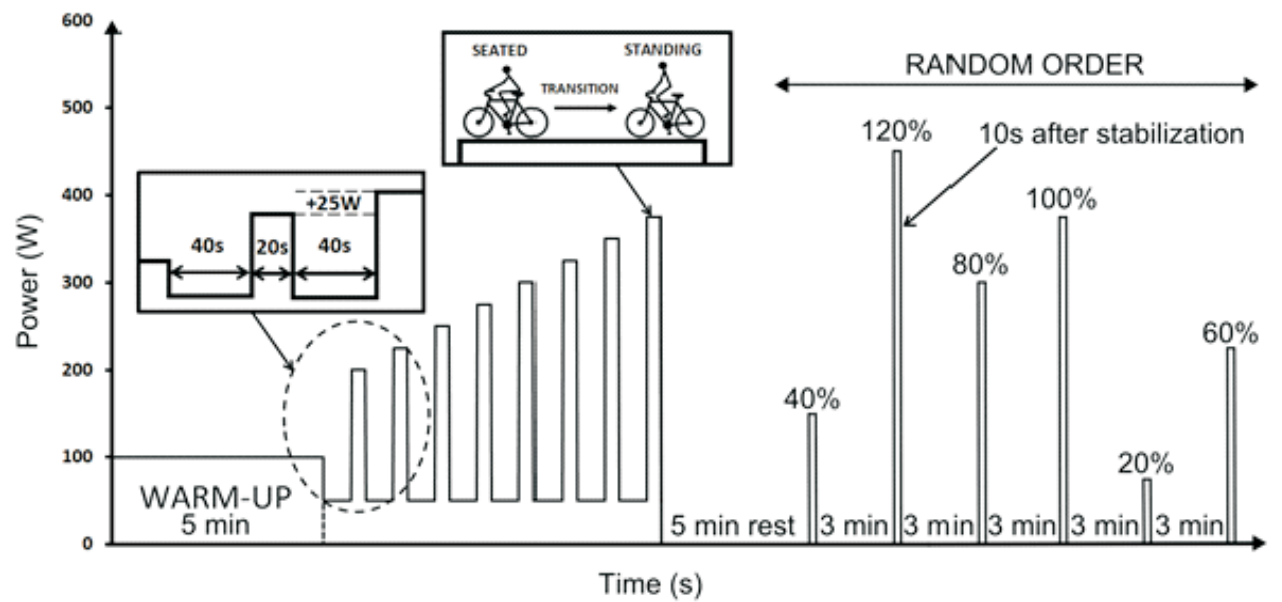

\section{Substracting weight \\ D Adding weight}
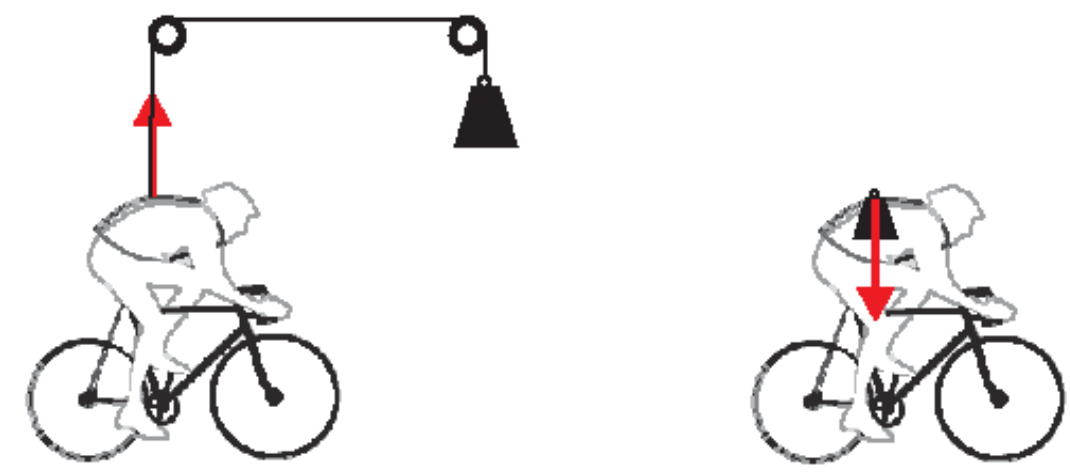

Figure 1

Fig. 1. Protocol description. A) Each subject performed five distinct sessions in random order. During three sessions, bodyweight (BW) was not modified $(100 \% \mathrm{BW})$ but cadence was modified (i.e., $=50,70$, or 90 RPM, with boxes arranged horizontally). During three sessions, the cadence was 70 RPM but weight was modified (i.e., $80 \%, 100 \%$, and $120 \%$ BW, with boxes arranged vertically). B) Illustration of protocol for one session. $\mathbf{C}$ and $\mathbf{D}$ ) Illustrations of the mechanisms used to modify the subject's weight. 

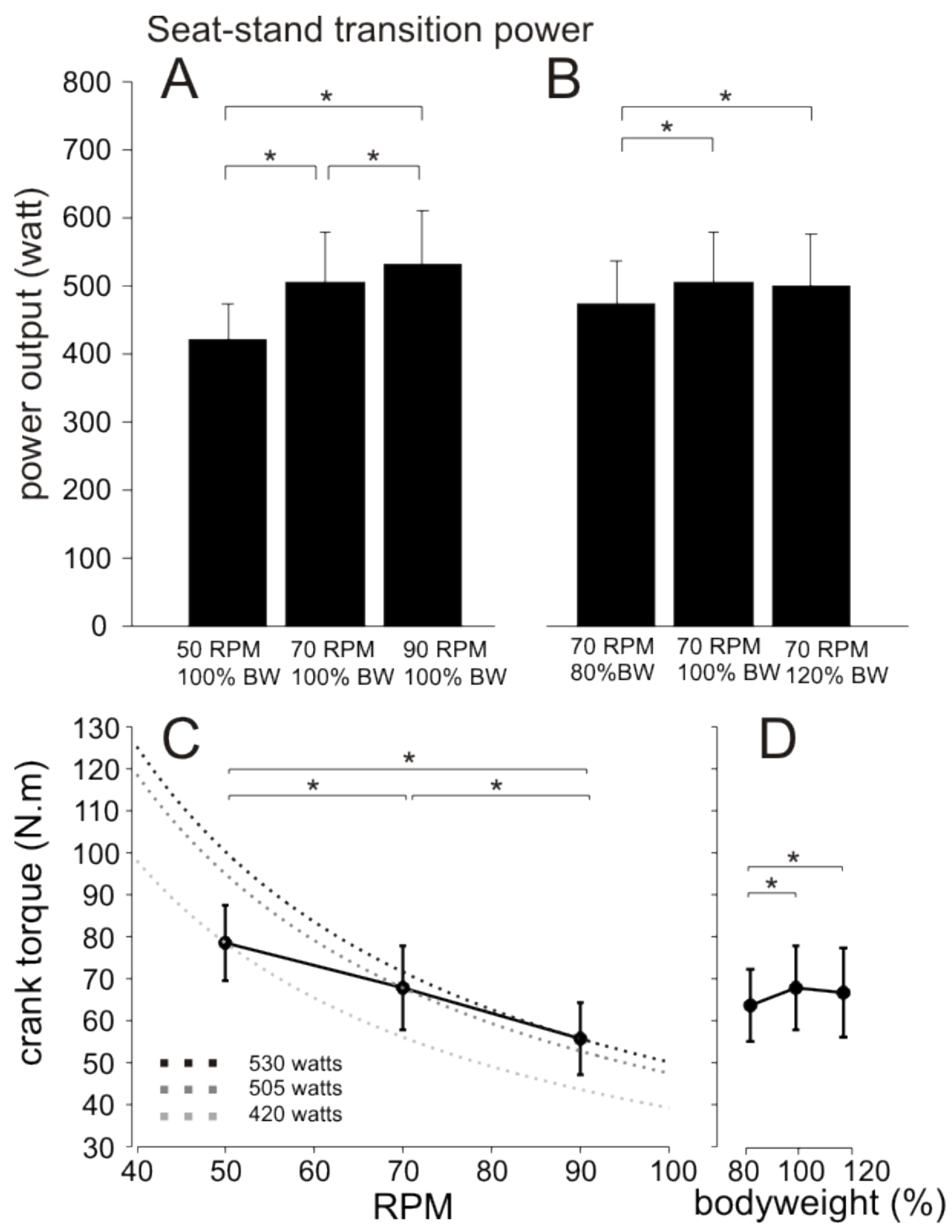

Figure 2

Fig. 2. SSTP values (group data). Effects of modifying cadence (A) or bodyweight (B) on the spontaneous seat-to-stand transition power (SSTP). SSTP corresponds to the power output at which the subject spontaneously used the standing position during incremental testing (i.e., test \#1 - see Fig. 1B). C) Relationships between crank-torque and cadence at SSTP in the $100 \%$ BW conditions. The dotted lines indicate power isolines. D) Crank-torque at $80 \%, 100 \%$ and 120\% BW. *: significant difference $(\mathrm{p}<0.05)$. 

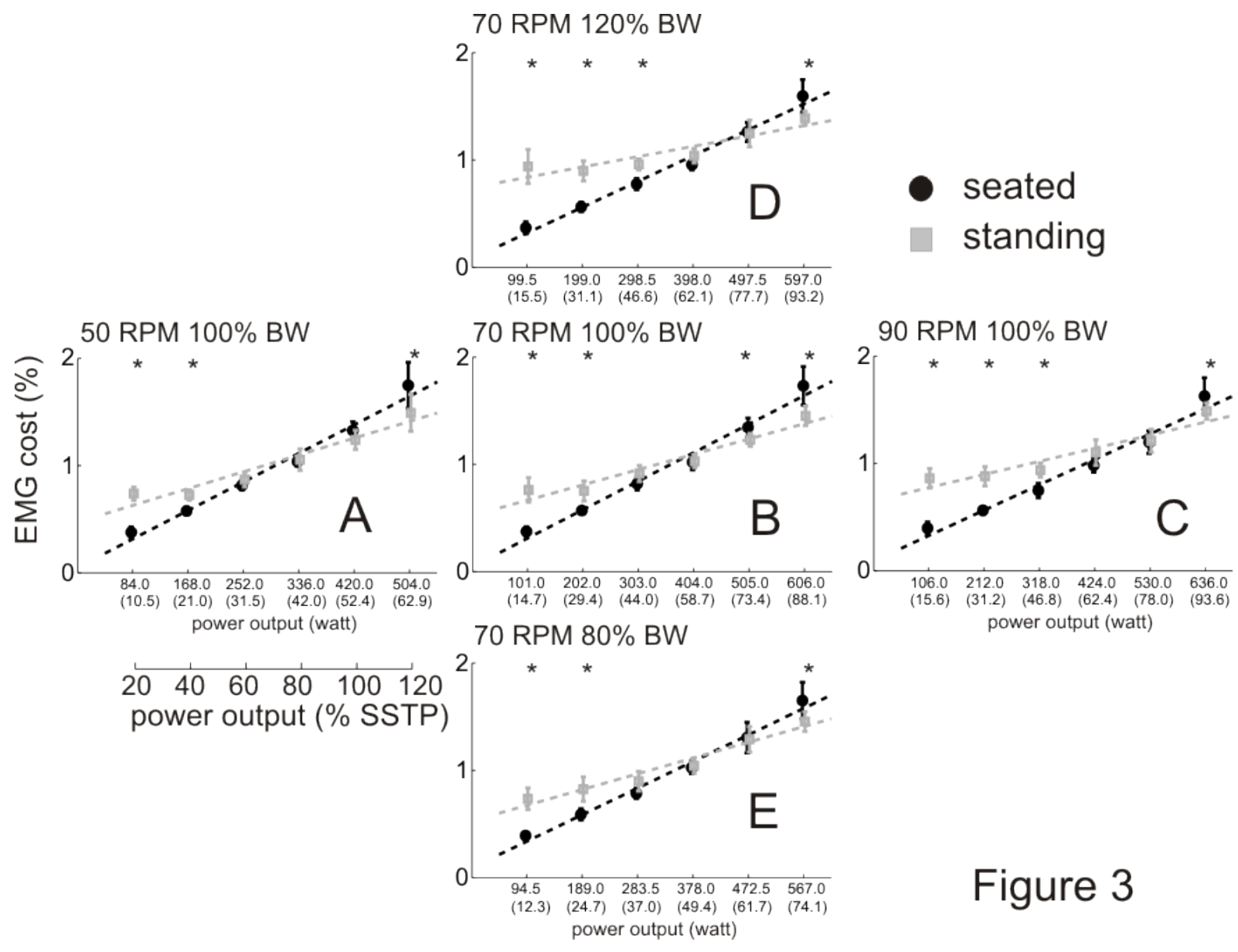

Fig. 3. EMG cost function (group data). Each subfigure (A to E) represents the EMG costs computed for a single session. The EMG cost is given as a \% of the mean EMG cost, as calculated from all the conditions presented during test \#2 (i.e., seated and standing at $20 \%$ to $120 \%$ of SSTP). The power outputs in the abscissa are given as a mean (SD) and correspond to $20 \%$ to $120 \%$ of SSTP. *: significant difference between the EMG cost in seated vs. standing position $(\mathrm{p}<0.05)$. 


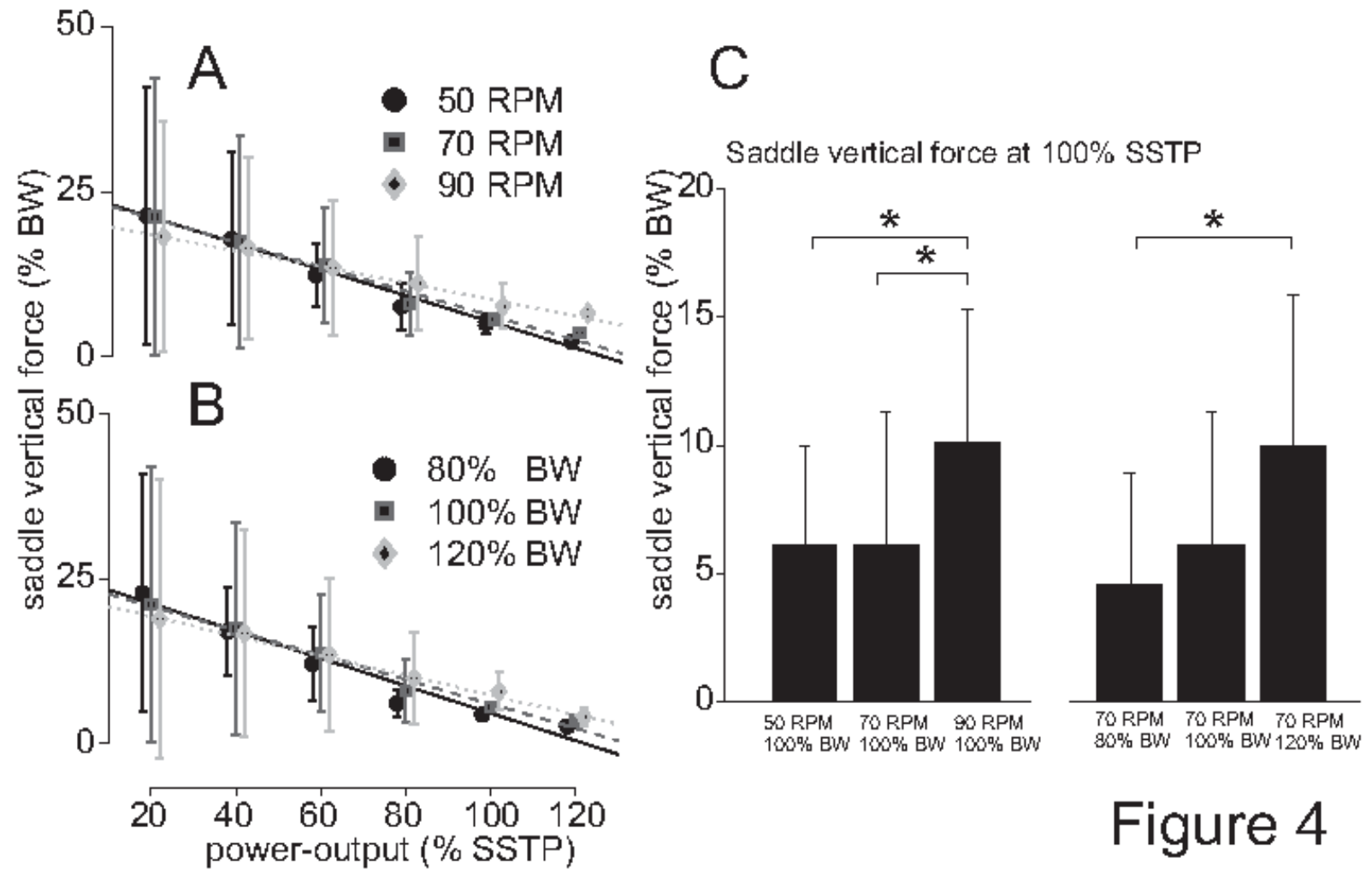

Fig. 4. Normalized saddle vertical forces. Effect of cadence (A) and bodyweight (B) on the minimal vertical saddle forces. Saddle forces are given as a \% of the subject's actual bodyweight (BW-see methods). C) Minimum saddle force values at $100 \%$ SSTP for each experimental condition. *: significant difference $(\mathrm{p}<0.05)$. 